\title{
POIKILOCYTOSIS IN THE ACNE VULGARIS COMORBIDITY WITH DEMODICOSIS AND FUNCTIONAL GALLBLADDER DISORDER
}

DOI: 10.36740/WLek202002115

\author{
Oksana K. Melekhovets, Alevtina S. Radko, Victor F. Orlovskiy, luirii V. Melekhovets, Tetiana 0. Kharchenko \\ SUMY STATE UNIVERSITY, SUMY, UKRAINE
}

\begin{abstract}
The aim of the study was to evaluate the role of poikilocytosis in the acne vulgaris comorbidity with demodicosis and functional gallbladder disorder. Materials and methods: The study included 140 people with a diagnosis of moderate acne: group 1 - patients with AV, group 2 - patients with AV + Demodicosis, group 3- AV + Demodicosis + functional gallbladder disorder (FGD). Biochemical analysis of lipid profile and liver enzymes, bilirubin was performed with semi-automatic analyzer HUMALYZER 3000. Scanning electron microscopy was performed using scanning electron microscope PEl-106I "SELMI”. Ultrasound investigation was carried out by the SonoScape S6Pro US-diagnostic system with $3-5$ MHz Ultrasound Probe Transducer C354.

Results: The most significant changes in blood erythrocytes morphology were observed in patients with comorbidity of acne, demodicosis and FGD with highest increasing of deformed erythrocytes up to $21 \%$, mainly due to echinocytes I, II order. Correlation analysis has revealed a strong direct association $r=+0.75$ ( $p<0.05)$ between the poikilocytosis and comorbidity of acne vulgaris with demodicosis and FGD.

Conclusions: A strong direct association between acne vulgaris comorbid pathology and the increased number of deformation shapes of erythrocytes confirms the impact of FGD on morphological characteristics of erythrocytes and developing of poikilocytosis.
\end{abstract}

KEY WORDS: acne vulgaris, Demodex, demodicosis, functional gallbladder disorders, red blood cells, scanning electron microscopy

Wiad Lek. 2020;73(2):289-292

\section{INTRODUCTION}

According to modern researchers, prevalence of acne vulgaris (AV) disease in puberty is $85 \%$ [1]. One of the basic pathogenetic mechanisms of the acne formation is an excess sebum production with following chemical composition change. Activation of pathogenic adaptive mechanisms leads to vasodilatation with stroma swelling and perivascular infiltrate following by intensive capillary growth. Endogenous and exogenous factors, such as $\mathrm{pH}$, osmolality, and others may affect red blood cells (RBC) morphology.

Erythrocytes are the main cellular component of the circulating blood. Mechanical properties of erythrocytes are associated with their ability for initial deformation, i.e. the capacity for shape change under applied forces which make it come in close contact with the capillary walls and, provide an adequate gas exchange. Morphologic aberrations (qualitative abnormalities) are highly relevant in clinical evaluation of red cell diseases [2].

Most abnormal red blood cells retain the ability to turn back into a discocyte when strength is removed. Such a transformed shape includes, foremost, echinocyte (erythrocyte with excrescences). Depending on the significance of crenation, echinocytes of types I, II, and III are distinguished. Normally, red blood cells of any abnormal shape and size account up 10\%. Poikilocytosis is the abnormal variation (more than 10\%) in shape of red blood cells [3].

Modern research in the field of skin diseases indicate high prevalence of acne vulgaris comorbidity with Demo- dex mites, gastrointestinal disorders, dishormonal states and others [4]. Demodex mites are found in $17-72 \%$ of healthy population of different age groups. The role of Demodex mites in the development and progression of acne has been attributed to the variety of pathogenic factors: changes in sebum production, induction of follicular hyperkeratosis, maintenance of local inflammation, effects on the immune system, which reduce the barrier function of the skin and facilitate microflora colonization.

Functional disorders of the gallbladder have a significant impact on the formation of acne vulgaris, as confirmed by detection FGD in $10-20 \%$ of young patients without somatic pathology [5].

\section{THE AIM}

The aim of the study was to evaluate the role of poikilocytosis in the acne vulgaris comorbidity with demodicosis and functional gallbladder disorder.

\section{MATERIALS AND METHODS}

A total of 140 patients with acne vulgaris were included. Patients with AV, comparable by age and sex (age of patients was $23 \pm 3.3$ years; male/female ratio was $43.5 / 56.5$ ), were divided into 3 groups based on the presents of comorbidity: group $1-40$ patients with AV; group $2(n=50)$ - comor- 
bidity of AV + demodicosis; group $3(\mathrm{n}=50)$ - comorbidity AV with demodicosis and FGD.

Biochemical analysis of lipid profile and liver enzymes, bilirubin was performed with semi-automatic analyzer HUMALYZER 3000. In advance of the study, all patients were recommended to stick to a low-fat and low-carb diet and also abstain from alcohol.

To investigate the possible association between erythrocyte's morphological characteristic and development of acne vulgaris in comorbid condition with demodicosis and FGD, scanning electron microscopy was performed in 50 patients of the study groups: 10 patients of the lgroup (control), 20 patients of the 2 group, 20 patients of the 3 group.

Studies on erythrocyte morphology were conducted by using the Romanovsky- Giemsa method with May-Grunwald modification with cytological analysis of smear and also using scanning electron microscopy. Scanning electron microscopy was performed by using scanning electron microscope PEI-106I "SELMI" with low-vacuum chamber and the possibility to make photos of the results obtained and store the data on computer storage device.

For the study was used $2 \mathrm{ml}$ of fasting blood from the cubital vein by adding 2 drops of sodium heparin. Samples were centrifuged for 5 minutes at $2000 \mathrm{rpm}$. The top fraction of centrifugate decanted, left $1 \mathrm{ml}$ and then fixed with an aldehyde fixative, using phosphate buffered saline 60 min exposure period. After the repeated centrifugation (2000 rpm, 5 minutes) the fixer was changed, and the centrifugation with the same parameters was performed again. Next step involved the preparation of erythrocyte washout from the fixative with the addition of phosphate-buffered saline. The resulting suspension was filtered through a series of increasing concentrations of ethanol and centrifugation performed after each stage. After treatment with $100 \%$ ethanol, final suspension and graphite spraying was carried out for further analysis by a scanning electron microscope.

Diagnosis of functional gallbladder disorder was established using Rome III Consensus (2006) criteria (which includes episodes of pain, dyspeptic disorders and early satiety or postprandial fullness and gallbladder ejection fraction $<40 \%$ [6]. The absence of significant changes in lipid metabolism and hepatic cytolysis parameters is one of the criteria for establishing a diagnosis of functional gallbladder disorder [7].

For assessing the gallbladder function, all patients had undergone abdominal ultrasound investigation using SonoScape S6Pro ultrasound diagnostic system with $3-5$ $\mathrm{MHz}$ Ultrasound Probe Transducer C354. To determine the functional disorder type, measurement of the gallbladder size and volume was performed with the calculating of the gallbladder ejection fraction (EF) according to the following formula:

$\mathrm{EF}=(1-\mathrm{RV} / \mathrm{FV}) \times 100$,

$\mathrm{RV}$ - residual volume of the GB; FV - fasting volume of the GB [10].

Ejection fraction values from 40 to $65 \%$ was used as normal range according to Rome III criteria.
Statistical processing of the obtained results was performed by using Windows 10 - Office Professional Plus software (Agreement ID: V0731528) with the use of parametric and non-parametric methods of variation statistics. Shapiro-Wilk test was applied to test normality of parameters. Data of lab analysis and morphological characteristics were represented as mean \pm SD values. Student's t-test with two-tailed distribution was used in statistical testing to determine the significance of differences between groups with AV; AV with demodicosis; AV with demodicosis and functional gallbladder disorders at 0.05 level of significance. Relative changes method was used to compare results in study groups. Pearson's correlation was used to calculate the correlation coefficient between poikilocytosis and comorbidity of AV with demodicosis and functional gallbladder disorders at 0.05 level of significance

\section{RESULTS AND DISCUSSION}

Baseline biochemical analysis showed that in all the groups biochemical hepatic indicators were in the normal range (tab. I), but in the group 3 an increasing in average concentrations of lipid metabolism indicators (TC by 13\%, LDL - C by $39 \%$ ) and liver tests (GGT by $22 \%$, ALT by $14 \%$, AST by $13,6 \%$ ) was detected.

The mean gallbladder ejection fraction of patients in group 3 at the time of enrollment was $34 \% \pm 4.6$, that confirmed hypomotor disorder of gallbladder. Groups 1 and 2 was characterized by normal average EF $-52 \pm 3.8$.

Scanning electron microscopy of peripheral blood smears demonstrated significant differences in erythrocytes morphology shift between the groups (tab.II).

The erythrocytes morphology deviations in patients of the group 1 (control) were less $10 \%$, what may be estimated as a normal value (fig. 1). Mild degree of erythrocytes abnormality was detected in the $2^{\text {nd }}$ group: increase in echinocytes by $20,9 \%$ and reduction in discocytes by $2.25 \%$ (fig. 2).

The most significant changes in blood erythrocytes morphology were observed in patients with comorbidity of acne, demodicosis and functional disorders of the hepatobiliary system.

Analysis of blood smear in the group 3 (fig. 3) demonstrated the discocytes reduction by $13.3 \%$, the echinocytes increase by $153 \%$ and irreversible shapes increase by 287 $\%$ compared to the values in the group 1 .

Pearson's correlation analysis revealed a strong direct association $\mathrm{r}=+0.75(\mathrm{p}<0.05)$ between the poikilocytosis and comorbidity of acne vulgaris combined with demodicosis and functional gallbladder disorder.

Presence of functional gallbladder disorder in the group 3 indicated the increase of deformed erythrocytes number up to $21 \%$, mainly due to echinocytes I, II order. Echinocytosis, somatocytosis and magnification of irreversible forms among patients in the group 3, vs the absence of these disorders in patients of groups 2 and 1, indicated a significant effect of somatic pathology on the quantitative and qualitative composition of red blood cells in the peripheral blood. 
Table I. Baseline data analysis across groups

\begin{tabular}{|c|c|c|c|}
\hline Characteristic & 1 group $(n=10)$ & 2 group $(n=20)$ & 3 group $(n=20)$ \\
\hline $\mathrm{TC}, \mathrm{mmol} / \mathrm{l}$ & $3,6 \pm 0,91^{*}$ & $3,96 \pm 1,1^{*}$ & $4,14 \pm 1,23^{* *}$ \\
\hline LDL - C, mmol/l & $1,9 \pm 0,86^{*}$ & $2,1 \pm 1,05^{*}$ & $3,14 \pm 0,98^{* *}$ \\
\hline VLDL - C, $\mathrm{mmol} / \mathrm{l}$ & $0,23 \pm 0,42$ & $0,34 \pm 0,44$ & $0,53 \pm 0,49$ \\
\hline $\mathrm{HDL}-\mathrm{C}, \mathrm{mmol} / \mathrm{l}$ & $1,32 \pm 0,21$ & $1,25 \pm 0,34$ & $1,1 \pm 0,39$ \\
\hline Atherogenic index & $2,2 \pm 1,89^{*}$ & $2,6 \pm 1,9^{*}$ & $3,1 \pm 1,68^{* *}$ \\
\hline Bilirubin, $\mu \mathrm{mol} / \mathrm{I}$ & $16,9 \pm 2,46$ & $18 \pm 1,34$ & $20,61 \pm 4,01$ \\
\hline GGT, U/I & $26,05 \pm 5,3^{*}$ & $28,6 \pm 6,04^{*}$ & $33,7 \pm 6,46^{* *}$ \\
\hline$A P, U / I$ & $72,2 \pm 12,2$ & $74,2 \pm 14,1$ & $75,3 \pm 15,3$ \\
\hline ALT, U/I & $18,3 \pm 9,1^{*}$ & $19,6 \pm 10,2^{*}$ & $21,2 \pm 11,4^{*}$ \\
\hline AST, U/I & $19,1 \pm 8,7^{*}$ & $20,4 \pm 9,3^{*}$ & $22,1 \pm 9,8^{* *}$ \\
\hline
\end{tabular}

${ }^{*} p>0,05 ;{ }^{* *} p<0,005$

TC - Total cholesterol,

LDL-C - Low-density lipoprotein cholesterol,

VLDL - C - very low-density lipoprotein cholesterol,

HDL-C - high-density lipoprotein cholesterol,

Al - atherogenic index,

GGT - gamma-Glutamyl Transferase,

$A P$ - alkaline phosphatase,

ALT alanine aminotransferase,

AST - aspartate aminotransferase.

Table II. The morphological characteristic of peripheral blood smear

\begin{tabular}{|c|c|c|c|c|c|c|}
\hline $\begin{array}{l}\text { Eritrocytes } \\
\text { morphology }\end{array}$ & $\begin{array}{c}1 \text { group } \\
(n=10)\end{array}$ & $\begin{array}{l}2 \text { group } \\
(n=20)\end{array}$ & 3 group $(n=20)$ & $\Delta_{1-2} \%$ & $\Delta_{1-3} \%$ & $\Delta_{2-3} \%$ \\
\hline Discocytes, \% & $90,43 \pm 4,3$ & $88,4 \pm 3,6$ & $78,4 \pm 7,6$ & 2,25 & 13,3 & 11,3 \\
\hline Echinocytes, \% & $7,04 \pm 3,4$ & $8,51 \pm 2,19$ & $17,84 \pm 8,94$ & 20,9 & 153 & 109,7 \\
\hline Stomatocytes,\% & $2,27 \pm 1,27$ & $2,68 \pm 1,32$ & $3,44 \pm 1,46$ & 18 & 51,5 & 28,3 \\
\hline Irreversible form, \% & $0,32 \pm 0,28$ & $0,41 \pm 0,29$ & $1,24 \pm 0,46$ & 28,1 & 287 & 202 \\
\hline
\end{tabular}

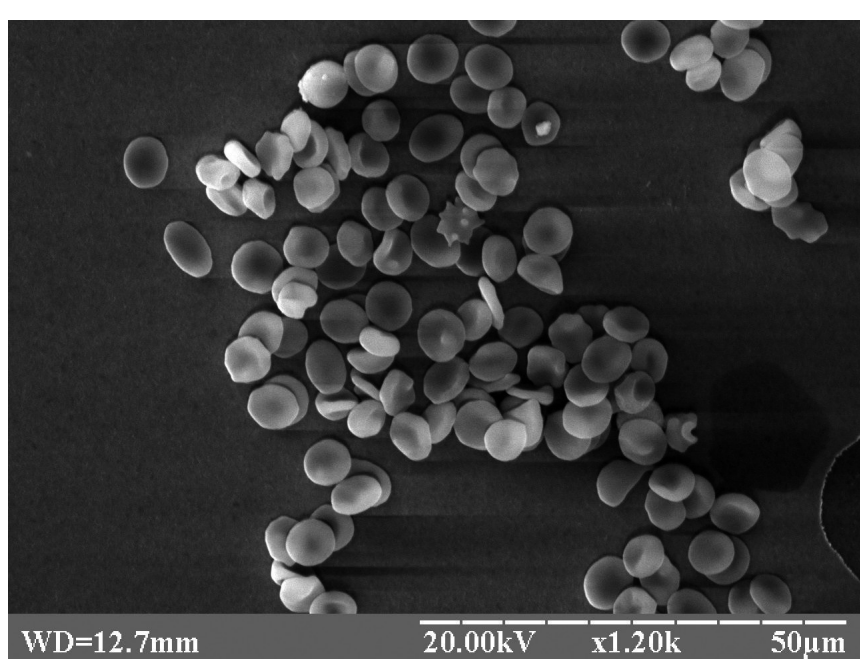

Fig. 1. Scanning electron microscope (SEM) images of the blood smear of the 1st group patient (AV): poikilocytosis 11,2\% (Original magnification 1200; scale bar $=50 \mu \mathrm{m})$.

It should be noted that changes in the morphology of red blood cells in patients of the group 3 , revealed by scanning electron microscopy, have the same pattern as described

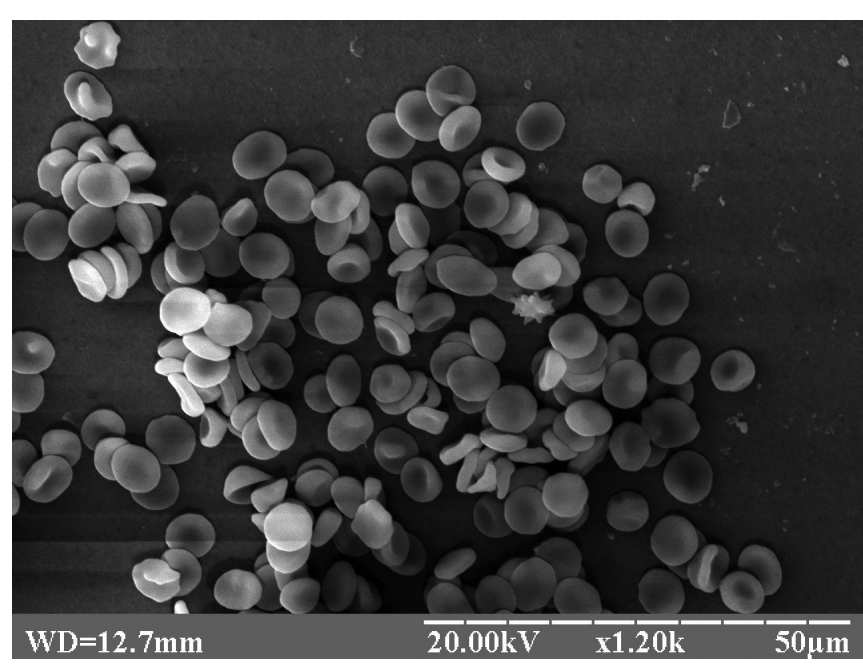

Fig. 2. Scanning electron microscope (SEM) images of the blood smear of the 2 nd group patient (AV + Demodicosis): poikilocytosis 11,2\% (Original magnification 1200; scale bar $=50 \mu \mathrm{m})$.

in the literature [8].

Disruption of microcirculation in $\mathrm{AV}$, associated with a decrease in erythrocyte deformability, significantly affects 


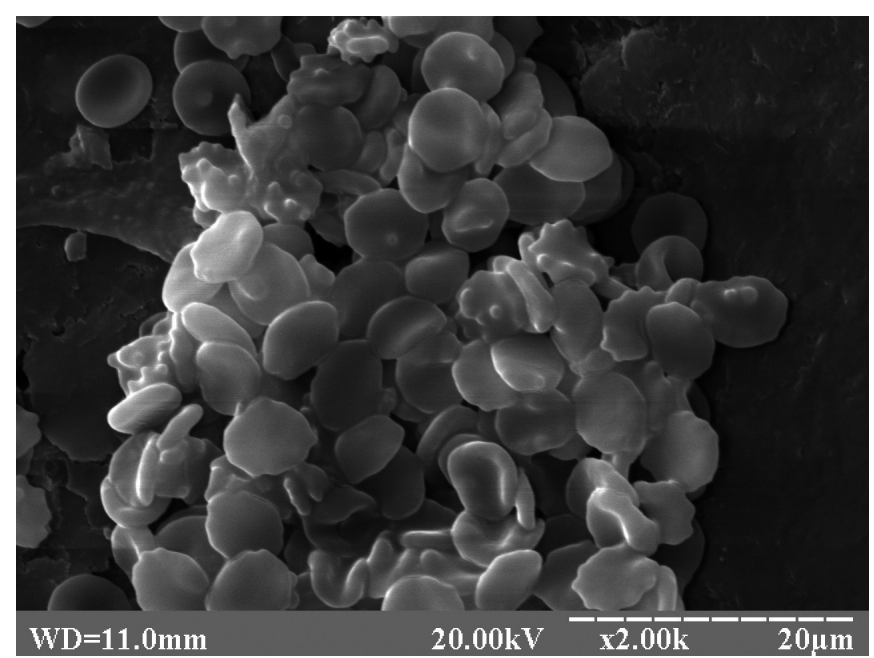

Fig. 3. Scanning electron microscope (SEM) images of the blood smear of the 3rd group patient (AV + Demodicosis + FDGBS): poikilocytosis $18.6 \%$. (Original magnification 2,000; scale bar $=20 \mu \mathrm{m}$ ).

microcirculatory blood flow, when red blood cells are forced to pass through small blood vessels (skin capillary), which causes tissue hypoxia [9].

Therefore, further studies are required to optimize acne treatment regimens, which should include adequate management for comorbid somatic pathology.

\section{CONCLUSIONS}

A strong direct association between the concomitant pathology of acne vulgaris and an increase in the number of initial forms of erythrocyte deformation confirms the influence of functional gallbladder disorder on the morphological characteristics of red blood cells and the occurrence of poikilocytosis. An increase in the number of pathological forms of erythrocytes under conditions of comorbidity leads to the disorders of microcirculation, the progression of inflammation, the persistence of microbiota and Demodex, which significantly declines the prognosis of the disease.

\section{REFERENCES}

1. Kutasevich YF, Oleinik IA, Mashtakova IA, V. Mangusheva Yu, Bronova IM. Comorbid pathology in a group of patients with moderate severity of acne. J of Dermatology and Cosmetology n. M0 Torsueva. 2014. 1-2 (32): 133.

2. Ademola Samson Adewoyin, Oluwafemi Adeyemi, Nosimot Omolola Davies and Ann Abiola Ogbenna (June 14th 2019). Erythrocyte Morphology and Its Disorders [Online First], Intech0pen, D0I: 10.5772/ intechopen.86112. Available from: https://www.intechopen.com/ online-first/erythrocyte-morphology-and-its-disorders.
3. Zohra et al. Evaluation of Severity in Patients of Acne Vulgaris by Global Acne Grading System in Bangladesh. Clin Pathol 2017; 1(1): 000105.

4. Litwin D, Chen W, Dzika E, Korycińska J. Human Permanent Ectoparasites; Recent Advances on Biology and Clinical Significance of Demodex Mites: Narrative Review Article. Iran J Parasitol. 2017;12(1):12-21.

5. Cotton P, Elta G, Carter R,Pasricha P,. Corazziari E. Gallbladder and Sphincter of Oddi Disorders. Gastroenterology 2016;150(6):1420-1429.

6. Donen A, Kantor R. Fatty meal ultrasonography in chronic acalculous cholecystitis. J Surg Case Rep. 2014(11):120. doi:10.1093/jscr/rju120.

7. Salem Awami. Evaluation of Gallbladder Motilityin Patients with Functional Gallbladder Disorder. Al-Mukhtar Journal of Sciences. 2018;33 (3)177-184.

8. Baybekov IM, Butaev AK, Khashimov FF, Mardonov DN, Baybekov Al. Healing of experimental wounds under influence of complex irradiation of light emitting diode of apparatus «barva-flex/bir». J Photobiology and Photomedicine. 2013; 1(2) 199-22.(Ukraine).

9. Grygorczyk1 R, Orlov S. Effects of Hypoxia on Erythrocyte Membrane Properties. Implications for Intravascular Hemolysis and Purinergic Control of Blood Flow. Front Physiol. 2017; 8: 1110. doi: 10.3389/ fphys.2017.01110.

\section{ORCID and contributionship:}

Oksana K. Melekhovets - 0000-0001-9031-7009 A,B,D,F

Alevtina S. Radko - 0000-0002-7673-9595 ${ }^{B, F}$

Iuirii V. Melekhovets - 0000-0002-3219-9021 E,F

Victor F. Orlovskiy - 0000-00025951-5047,,$F$

Tetiana O. Kharchenko - 0000-0002-7690-2954 ${ }^{E, F}$

\section{Conflicts of interest:}

Authors declare no conflict of interest.

\section{CORRESPONDING AUTHOR \\ Oksana K. Melekhovets}

Sumy State University

1 Sanatorna Str., 40018, Sumy, Ukraine

tel: +380667122929

e-mail:melekhovets.oksana@gmail.com

Received: 08.09 .2019

Accepted: 17.12.2019

A - Work concept and design, B - Data collection and analysis, C - Responsibility for statistical analysis, D-Writing the article, $\mathbf{E}$-Critical review, $\mathbf{F}$ - Final approval of the article 